\title{
Do outlier inpatients experience more emergency calls in hospital? An observational cohort study
}

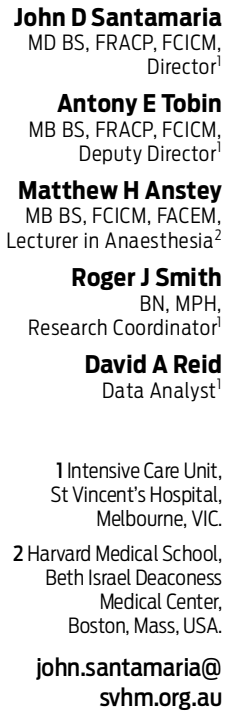

MJA 2014; 200: 45-48 doi: 10.5694/mjal2.11680 s medicine has become more specialised, so has the delivery of care in hospitals. For example, orthopaedic patients are separated from those who have had a stroke, and those with cardiac disease are separated from surgical patients. This model of care, where medical specialists, allied health staff and nurses work together in teams, builds expertise and improves patient care. ${ }^{1-3}$ However, competing with this model is increasing pressure on hospital beds. In Australia between 2006-07 and 2010-11, hospital separations increased by about $4 \%$ per year and emergency department presentations by $3 \%$, while bed numbers increased by only $1 \%{ }^{4}$ This could indicate that hospitals have become more efficient; however, in Victoria, waiting lists have increased while patient throughput remains unchanged. ${ }^{5}$ With pressure to move patients out of the emergency department (eg, through the National Emergency Access Target) comes pressure to place patients in a bed on a ward, ${ }^{6}$ which may not be the appropriate ward to provide specialised care.

System-related factors that appear to influence mortality include prolonged waits for admission from the emergency department, the volume of patients undergoing a certain procedure in a hospital, and night-time discharge of patients from the intensive care unit. $^{7-9}$ Similarly, there may be an optimal location in the hospital to receive care, where the staff have particular skills in treating the patient's condition and are more familiar with signs of deterioration. Clinical deterioration occurs due to disease severity or to inadequate or inappropriate care. ${ }^{10}$ Inadequate care may mean that the skills of the personnel in the unit are insufficient for the patient. Thus, the location of a patient in a "home" ward or an "outlier" (non-home) ward may influence the patient's outcome.

The use of rapid response or medical emergency teams (METs) to

\begin{abstract}
Objective: To determine the effect of spending time as an outlier (ie, an inpatient who spends time away from his or her "home" ward) on the frequency of emergency calls for patients admitted to a tertiary referral hospital.
\end{abstract}

Design, setting and patients: Observational cohort study of all patients admitted to a university-affiliated tertiary referral hospital in Melbourne, Victoria, between 1 July 2009 and 30 November 2011.

Main outcome measure: The number of emergency calls per hospital admission, with reference to location within the hospital.

Results: There were 58158 admissions during the study period. The median age of admitted patients was 61 years, $55 \%$ were male, and the inhospital mortality was $1.40 \%$. In 11034 admissions (18.97\%), patients spent time as outliers. Inhospital mortality was $2.57 \%$ in the outlier group versus $1.12 \%$ in the nonoutlier group $(P<0.001)$. After adjusting for age, same-day admission, 10 -year predicted mortality, interhospital transfer and high-risk clinical units, outlier status was associated with a 53\% increase in emergency calls $(P<0.001)$.

Conclusions: This study found a strong association between time spent away from a patient's home ward and the number of emergency calls. We postulate that outlier patients are at risk as they may have therapeutic and monitoring needs that are only available on their home ward. With increasing pressure to move patients out of the emergency department, the number of outlier patients may increase.

respond promptly to clinical deterioration and reduce inhospital adverse events has significant appeal, although strong evidence regarding effectiveness is lacking. ${ }^{11}$ However, such teams may be treating the result of failure to monitor or to escalate care $^{12}$ rather than the root cause. Research has suggested that medical patients on non-medical wards are more likely to require MET activation, ${ }^{13}$ implying that MET activation may be a marker of quality of ward care. Using MET activation as a marker for clinical deterioration or adverse events, we sought to investigate the relationship between patient outlier status and number of emergency (MET) calls.

\section{Methods}

St Vincent's Hospital Melbourne in Victoria is a 400-bed, university-affiliated tertiary referral hospital. This study was approved as a quality assurance activity by the hospital's human research ethics committee.

All patients admitted between 1 July 2009 and 30 November 2011 were eligible. We excluded a small number of patients who were admitted for outpatient testing (eg, endocrine testing, overnight polysomnography), mental health care, rehabilitation or palliative care. Home wards were designated as general medical or surgical.

Clinical services in the hospital are broadly divided into medicine and surgery, with specialised units such as cardiac surgery, cardiology and gastroenterology. The services are supported by a busy emergency department, 15-bed intensive care unit, and 12 operating theatres. The inpatient building has seven floors dedicated to patient care. The allocation of units to dedicated wards was standardised several years ago and remained stable during the study period. Each patient admitted to the hospital was allocated to a medical or surgical unit. Admissions that took place under medical services but were later transferred to surgical services are included with the latter.

The hospital's MET was established in 2002 as part of the MERIT study ${ }^{14}$ but continued after the study's completion. The team comprises an intensive care registrar or Fellow, intensive care nurse and general medical regis- 
1 Characteristics of patients admitted to hospital, 1 July 2009 - 30 November 2011

\begin{tabular}{|c|c|c|c|c|}
\hline & \multirow[b]{2}{*}{ All admissions } & \multicolumn{3}{|c|}{ Admissions by outlier status } \\
\hline & & Non-outlier & Outlier & $P$ \\
\hline Number of admissions* & 58158 & 47124 & 11034 & \\
\hline Age in years, median (IQR) & $61.3(44.1-74.5)$ & $60.8(44.0-73.5)$ & $64.0(44.3-78.0)$ & $<0.001$ \\
\hline Male & $31984(55.00 \%)$ & $25819(54.79 \%)$ & $6165(55.87 \%)$ & 0.02 \\
\hline Admission origin & & & & $<0.001$ \\
\hline Home & $53455(91.91 \%)$ & $43154(91.58 \%)$ & $10301(93.36 \%)$ & \\
\hline Interhospital transfer & $4226(7.27 \%)$ & $3652(7.75 \%)$ & $574(5.20 \%)$ & \\
\hline Residential aged care & $477(0.82 \%)$ & $318(0.67 \%)$ & $159(1.44 \%)$ & \\
\hline Hospital length of stay in days, median (IQR) & $1.17(0.30-4.79)$ & $0.85(0.13-3.33)$ & $4.17(2.00-8.59)$ & $<0.001$ \\
\hline Same-day admission & $20029(34.44 \%)$ & $19599(41.59 \%)$ & $430(3.90 \%)$ & $<0.001$ \\
\hline Emergency admission & $28875(49.65 \%)$ & $19934(42.30 \%)$ & $8941(81.03 \%)$ & $<0.001$ \\
\hline Predicted 10-year mortality, ${ }^{\dagger}$ median (IQR) & $10 \%(2 \%-47 \%)$ & $10 \%(2 \%-22 \%)$ & $22 \%(2 \%-47 \%)$ & $<0.001$ \\
\hline Number of complications & & & & $<0.001$ \\
\hline 0 & $46738(80.36 \%)$ & 38967 (82.69\%) & $7771(70.43 \%)$ & \\
\hline $1-10$ & $10835(18.63 \%)$ & $7742(16.43 \%)$ & $3093(28.03 \%)$ & \\
\hline $11-20$ & $513(0.88 \%)$ & $366(0.78 \%)$ & $147(1.33 \%)$ & \\
\hline $21-30$ & $62(0.11 \%)$ & $44(0.09 \%)$ & $18(0.16 \%)$ & \\
\hline $31-40$ & $10(0.02 \%)$ & $5(0.01 \%)$ & $5(0.05 \%)$ & \\
\hline Inhospital mortality & $812(1.40 \%)$ & $528(1.12 \%)$ & $284(2.57 \%)$ & $<0.001$ \\
\hline Medical patients & $34863(59.95 \%)$ & 28251 (59.95\%) & $6612(59.92 \%)$ & 0.97 \\
\hline Units with most emergency calls & & & & $<0.001$ \\
\hline Neurosurgery & $2798(4.81 \%)$ & $1875(3.98 \%)$ & $923(8.37 \%)$ & \\
\hline Cardiothoracic surgery & $1581(2.72 \%)$ & $1454(3.09 \%)$ & $127(1.15 \%)$ & \\
\hline General surgery & $3956(6.80 \%)$ & $3183(6.75 \%)$ & $773(7.01 \%)$ & \\
\hline Vascular surgery & $919(1.58 \%)$ & $664(1.41 \%)$ & $255(2.31 \%)$ & \\
\hline Orthopaedic surgery & $2726(4.69 \%)$ & $2247(4.77 \%)$ & $479(4.34 \%)$ & \\
\hline General medicine & $5155(8.86 \%)$ & $1987(4.22 \%)$ & $3168(28.71 \%)$ & \\
\hline Days as an outlier, median (IQR) & - & - & $0.82(0.23-2.06)$ & \\
\hline Percentage of admission as an outlier (IQR) & - & - & $24 \%(6 \%-81 \%)$ & \\
\hline
\end{tabular}

IQR = interquartile range. * Includes patients who were readmitted during the study period. † Using Charlson Comorbidity Index.

trar. The MET calling criteria are: respiratory rate $<5$ or $>36$ breaths $/ \mathrm{min}$, oxygen saturation measured by pulse oximetry $<90 \%$ despite supplemental oxygen, pulse rate $<40$ or $>140$ beats/ min, systolic blood pressure $<90 \mathrm{mmHg}$, unexpected deterioration in conscious state, repeated or prolonged seizures, or any patient about whom staff are sufficiently worried. Each emergency call is entered in a dedicated database and reviewed by intensive care physicians each week.

The hospital's patient master index (PAS; Healthcare Group, CSC) includes data on all admitted patients. In addition, to meet funding requirements in Victoria, up to 40 International Classification of Diseases, 10th revision, Australian modification (ICD-10-AM) diagnoses and 40 procedures are recorded. Each ICD-10AM code has a prefix indicating whether the condition was the primary diagnosis $(\mathrm{P})$ or a complication during admission $(\mathrm{C})$. The $\mathrm{C}$-prefix codes have been used to monitor hospital activity. ${ }^{15}$ We used these diagnostic codes with the Charlson Comorbidity Index to calculate predicted 10 -year mortality. ${ }^{16}$ The patient master index also lists all bed movements for each admission. Using the unit-ward allocations, we determined whether patients spent time outside their home ward and calculated the percentage of the admission spent as an outlier. Time spent in an intensive care or coronary care unit was not considered outlier time. Admissions were classified as same-day if the admission and discharge dates were the same.

\section{Statistical analysis}

Results are expressed as mean and standard deviation or median and interquartile range (IQR), depending on the variable's distribution. Categorical variables are reported as numbers and percentages. We compared continuous variables using the Mann-
Whitney $U$ test, and categorical variables with the Fisher exact or $\chi^{2}$ test. As some patients had more than one emergency call, we adjusted for confounding variables by modelling the data using zero-inflated negative binomial regression. ${ }^{17}$ Previous hospital audits had identified that patients in some clinical units (neurosurgery; cardiothoracic, vascular and abdominal surgery; nephrology; and general medicine) had more emergency calls than those in other units. We therefore included these high-risk clinical units in the multivariate analysis, along with variables found to be statistically significant in the univariate analysis. Data were analysed with Stata version 12 (StataCorp). Statistical significance was set at $P<0.05$.

\section{Results}

There were 58158 admissions during the study period. No adjustment was made for patients who were readmitted during the study. Demographic information is shown in Box 1. The median age was 61 years, with a slight preponderance of men. Most patients were admitted from home. Thirtyfour per cent of patients had a sameday admission, and half of all admissions were classified as emergency (28\% of same-day admissions). The predicted 10-year mortality rate for all admissions was $10 \%$. The actual inhospital mortality was $1.40 \%$. Most patients were admitted under medical units. There were $155 \mathrm{admissions}$ that initially took place under medical services, but were later transferred to surgical services.

In 11034 admissions (18.97\%), the patients spent time outside their home wards, with a median duration as an outlier of 0.82 days (Box 1). Patients spending time outside their home wards were older and had longer hospital stays, greater severity of illness (by the Charlson Comorbidity Index), more complications and higher mortality than those who did not. There were similar proportions of medical and surgical patients in the outlier and non-outlier groups, although outlier patients were more likely to be general medical or neurosurgical patients. Patients with sameday admissions were far more likely to be managed entirely on their home ward. 
Details of emergency calls are shown in Box 2. There were 1501 emergency calls made for 1115 patients, of which 93 calls (for 89 patients) were for cardiac arrest. Patients who had emergency calls had significantly higher mortality than those who did not $(25.2 \%$ [95\% CI, $22.6 \%-27.8 \%$ ] v $0.93 \%$ [ $95 \%$ CI, $0.85 \%-1.01 \%])$. The mortality for patients needing three or more calls was 39\%. Patients who experienced some period as an outlier were more likely to have an emergency call than those who did not $(3.8 \%$ [95\% CI, $3.5 \%-4.2 \%$ ] v $1.5 \%$ [ $95 \%$ CI, $1.4 \%-$ $1.6 \%])$. For the outlier group, 528 of 607 calls $(87.0 \%)$ occurred while the patients were away from their home ward. Patients in the outlier group were also more likely to have cardiac arrests.

After adjusting for high-risk clinical units, age, same-day admission, 10year predicted mortality, and interhospital transfer, spending time outside the home ward was associated with a $53 \%$ (95\% CI, 32\%-77\%) increase in emergency calls (Box 3).

As a higher proportion of patients in the outlier group were classified as emergency admissions, we separately estimated the outlier effect on emergency calls for emergency admissions (relative risk, 1.44; 95\% CI, 1.21-1.70) and elective admissions (relative risk, 2.04; 95\% CI, 1.49-2.80). While the relative risk was higher in the elective admission group, this difference did not achieve statistical significance.

\section{Discussion}

This study in an academic tertiary referral hospital found that patients cared for outside their home ward had more comorbidities, more emergency calls and worse hospital outcomes (mortality and complications) than those who remained on their home wards. After adjusting for relevant factors, spending time outside the home ward was associated with a $53 \%$ increase in emergency calls.

Clinical deterioration in hospital patients causes increased morbidity and mortality. ${ }^{18}$ Such deterioration may be due to the severity of the patient's disease (and therefore not preventable or expected), but in some cases it may result from a failure of the primary team to recognise and man-

\section{Distribution of emergency calls for patients, 1 July 2009 - 30 November 2011}

Admissions by outlier status

\begin{tabular}{lcccc} 
& All admissions & Non-outlier & Outlier & $P$ \\
\cline { 3 - 5 } Total number of emergency calls & 1501 & 894 & 607 & \\
Number of calls per patient & $n=58158$ & $n=47124$ & $n=11034$ & $<0.001$ \\
0 calls & $57043(98.08 \%)$ & $46432(98.53 \%)$ & $10611(96.17 \%)$ & \\
1 call & $858(1.48 \%)$ & $550(1.17 \%)$ & $308(2.79 \%)$ & \\
2 calls & $183(0.31 \%)$ & $103(0.22 \%)$ & $80(0.73 \%)$ & \\
$\geqslant 3$ calls & $74(0.13 \%)$ & $39(0.08 \%)$ & $35(0.32 \%)$ & \\
Cardiac arrests & $89(0.15 \%)$ & $56(0.12 \%)$ & $33(0.30 \%)$ & $<0.001$
\end{tabular}

age a worsening clinical situation. This might be due to a lack of expertise of nursing staff when faced with patients with unusual conditions (eg, heart failure patients on surgical wards), delayed calling of medical staff, or no available medical staff in the immediate vicinity. While METs were implemented to rescue patients from cardiac arrest caused by clinical deterioration, ${ }^{14}$ their activation might reflect a failure of the primary team to recognise and manage deterioration. There is evidence that home ward allocation may improve outcomes by offering specialised treatment, such as that provided by stroke management teams. ${ }^{19}$ Our study supports this hypothesis, as patients moved outside their home ward had more emergency calls and worse clinical outcomes.

To the best of our knowledge, this is the first study to document the extent of movement of patients from their home wards to other wards within a large hospital. These patients had well defined characteristics that would indicate a more complicated course, such as more comorbidities, multiday admissions and older age. They also had worse outcomes, with longer length of stay, more complications and higher mortality. This implies that in an environment where beds are limited, it makes sense to place patients at high risk of complications in the appropriate hospital location. If forced to choose which patients should be outliers, our study suggests that younger patients with same-day admissions are less likely to experience deterioration. Better solutions might include establishing short-stay surgical units for elective patients, short-stay wards for medical and surgical patients awaiting admission, liaison services for less well patients, and early referral of surgical patients to medical units.

Our study has some limitations. The analysis involved data from a single hospital and would benefit from external validation at other centres. While we had complete data on the location of each emergency call, we did not have details on the nature of the calls. For example, calls for a change in conscious state or hypoten-

\section{Results of multivariate analysis of the number of emergency calls and the associated relative risk for selected factors}

\begin{tabular}{lcl} 
& Relative risk $(95 \% \mathrm{Cl})$ & $P^{*}$ \\
\hline Age (per year of increase) & $0.988(0.979-0.997)$ & $<0.01$ \\
Any time outside home ward & $1.530(1.322-1.769)$ & $<0.001$ \\
Predicted mortality $(<46 \% \mathrm{v} \geqslant 46 \%)^{\dagger}$ & $1.826(1.322-2.522)$ & $<0.001$ \\
Interhospital transfer & $2.370(1.974-2.847)$ & $<0.001$ \\
Same-day admission & $0.022(0.011-0.042)$ & $<0.001$ \\
Neurosurgery unit & $2.262(1.784-2.868)$ & $<0.001$ \\
Cardiothoracic surgery unit & $2.136(1.585-2.878)$ & $<0.001$ \\
General surgery unit & $1.401(1.069-1.836)$ & $<0.05$ \\
Nephrology unit & $2.109(1.471-3.024)$ & $<0.001$ \\
General medicine unit & $2.010(1.684-2.400)$ & $<0.001$ \\
\hline
\end{tabular}

*From zero-inflated negative binomial regression. Although orthopaedic unit and emergency admission were significant on univariate analysis, they were not significant in the final multivariate model. † Predicted 10-year mortality based on Charlson Comorbidity Index is not a continuous variable and occurs in eight groups (eg, $1.7 \%, 4.1 \%$ ), simplified to two groups for this analysis. At a predicted mortality $\geqslant 46 \%$ (representing $27 \%$ of the cohort), there was a significant increase in actual inhospital mortality. 
sion have more significance than those for seizures or faints. ${ }^{20}$ Furthermore, we did not routinely record the period of instability before emergency calls to determine if the increased calling rate was due to less recognition of deterioration or delayed action by ward staff. However, while an increase in calls may have been due to unfamiliarity with outlier patients, the increased mortality and complications in these patients might suggest a lower quality of care. Potential confounders exist, particularly with respect to emergency status and same-day admission, but we included these in the multivariate analysis. Resuscitation status was not recorded.

It is unlikely that a randomised controlled trial of patient allocation to home or non-home wards will ever be undertaken, for logistical and ethical reasons. However, movement of patients between wards may be affected by administrative decisions. In Australia, the National Emergency Access Target has put pressure on hospitals to admit patients to a ward within a 4-hour limit. ${ }^{6}$ Furthermore, the nature of hospital funding tends to favour elective surgery, so hospitals may admit these patients to home wards and only repatriate patients with more complex conditions when beds are less constrained. Clinicians and administrators must ensure that targets or new processes are evaluated and continually monitored to ensure they produce benefit, not harm. While mortality is an uncommon but important measure of harm, changes in morbidity and even emergency call rates might be just as important and may draw attention to a harmful process before the mortality rate rises.
Health departments and clinicians have put greater focus on preventing and treating clinical deterioration. Solutions have included observation charts designed with human factor consideration, general ward electronic monitoring, and METs. While we recognise the success of METs in preventing inhospital deaths, we need to consider whether processes of care are placing patients at risk. More deterioration occurs when nurses have less time to observe patients, such as overnight or during drug rounds or clinical handover. ${ }^{13}$ Nursing ratios may also affect detection of deterioration, but there are conflicting data about this. ${ }^{21,22}$ We can now add the patient's outlier status to this list of variables. Bed management and bed allocation on the wards therefore need to be considered in programs aimed at improving care.

In summary, this study suggests that placement of patients within the hospital matters. If you have to be in a hospital, then the home ward is the place to be.

Competing interests: No relevant disclosures.

Received 15 Nov 2012, accepted 5 Sep 2013.

1 Grumbach K, Bodenheimer T. Can health care teams improve primary care practice? JAMA 2004; 291: 1246-1251.

2 Leape L, Berwick D, Clancy C, et al. Transforming healthcare: a safety imperative. Qual Saf Health Care 2009; 18: 424-428.

3 Wagner EH. The role of patient care teams in chronic disease management. BMJ 2000; 320: 569-572.

4 Australian Institute of Health and Welfare. Australian hospital statistics 2010-11. Canberra: AlHW, 2012. (AlHW Cat. No. HSE 117; Health Services Series No. 43.) http://www.aihw.gov.au/ publication-detail/?id=10737421633 (accessed Sep 2013).

5 Victorian Department of Health. Victorian health services performance report: December 2011 quarter. Melbourne: Department of Health, 2011. http://performance.health.vic.gov.au/Renderers/ ShowMedia.ashx? $\mathrm{id}=53 \mathrm{c} 42 \mathrm{a} 45-14 \mathrm{fb}-404 \mathrm{c}-$ 9005-074lebb5225f (accessed Aug 2013)
6 Australian Government Department of Health and Ageing. Hospitals. Shorter public hospital waiting times: emergency departments. http:// www.health.gov.au/internet/yourhealth/ publishing.nsf/Content/theme-hospitals (accessed Dec 2013).

7 Rogers SO Jr, Wolf RE, Zaslavsky AM, et al. Relation of surgeon and hospital volume to processes and outcomes of colorectal cancer surgery. Ann Surg 2006; 244: 1003-1011.

8 Sprivulis PC, Da Silva JA, Jacobs IG, et al. The association between hospital overcrowding and mortality among patients admitted via Western Australian emergency departments. Med J Aust 2006; 184: 208-212.

9 Tobin AE, Santamaria JD. After-hours discharges from intensive care are associated with increased mortality. Med J Aust 2006; 184: 334-337.

10 Litvak E, Pronovost PJ. Rethinking rapid response teams. JAMA 2010; 304: 1375-1376.

11 Chan PS, Jain R, Nallmothu BK, et al. Rapid response teams: a systematic review and metaanalysis. Arch Intern Med 2010; 170: 18-26.

12 Jones DA, DeVita MA, Bellomo R. Rapid-response teams. N Engl J Med 2011; 365: 139-146.

13 Schmid-Mazzoccoli A, Hoffman LA, WolfGA, et al. The use of medical emergency teams in medical and surgical patients: impact of patient, nurse and organisational characteristics. Qual Saf Health Care 2008; 17: 377-381.

14 Hillman K, Chen J, Cretikos M, et al; MERIT study investigators. Introduction of the medical emergency team (MET) system: a clusterrandomised controlled trial. Lancet 2005; 365: 2091-2097.

15 Ehsani JP, Jackson T, Duckett SJ. The incidence and cost of adverse events in Victorian hospitals 2003-04. Med J Aust 2006; 184: 551-555.

16 Charlson ME, Pompei P, Ales KL, MacKenzie CR. A new method of classifying prognostic comorbidity in longitudinal studies: development and validation. J Chronic Dis 1987; 40: 373-383.

17 Hilbe JM. Negative binomial regression. 2nd ed Cambridge: Cambridge University Press, 2011.

18 Jones DA, Dunbar NJ, Bellomo R. Clinical deterioration in hospital inpatients: the need for another paradigm shift. Med J Aust 2012; 196: 97-100.

19 Stroke Unit Trialists' Collaboration. Organised inpatient (stroke unit) care for stroke. Cochrane Database Syst Rev 2007; (4): CD000197.

20 Buist M, Bernard S, Nguyen TV, et al. Association between clinically abnormal observations and subsequent in-hospital mortality: a prospective study. Resuscitation 2004; 62: 137-141.

21 Aiken LH, Clarke SP, Sloane DM, et al. Hospital nurse staffing and patient mortality, nurse burnout, and job dissatisfaction. JAMA 2002; 288: 1987-1993.

22 Needleman J, Buerhaus P, Mattke S, et al. Nursestaffing levels and the quality of care in hospitals. NEngl J Med 2002; 346: 1715-1722. 